\title{
Reactive Oxygen Species in Anticancer Immunity: A Double-Edged Sword
}

\author{
Jie Wang ${ }^{1,2}$, Ning Liu $^{2}$, Hongfei Jiang ${ }^{1,2}$, Qian $L^{2 *}$ and Dongming Xing ${ }^{1,2,3 *}$ \\ ${ }^{1}$ The Affiliated Hospital of Qingdao University, Qingdao University, Qingdao, China, ${ }^{2}$ Qingdao Cancer Institute, Qingdao \\ University, Qingdao, China, ${ }^{3}$ School of Life Sciences, Tsinghua University, Beijing, China
}

Reactive oxygen species (ROS) are critical mediators in many physiological processes including innate and adaptive immunity, making the modulation of ROS level a powerful strategy to augment anticancer immunity. However, current evidences suggest the necessity of a deeper understanding of their multiple roles, which may vary with their concentration, location and the immune microenvironment they are in. Here, we have reviewed the reported effects of ROS on macrophage polarization, immune checkpoint

OPEN ACCESS

Edited by:

Yong Liu,

University of Chinese Academy of

Sciences, China

Reviewed by:

Yuce $L i$,

Sungkyunkwan University, South

Korea

Wancun Zhang,

Zhengzhou University, China

*Correspondence:

Qian Li

liqian123@qdu.edu.cn

Dongming Xing

xdm@qdu.edu.cn

Specialty section:

This article was submitted to Biomaterials,

a section of the journal

Frontiers in Bioengineering and

Biotechnology

Received: 28 September 2021

Accepted: 29 October 2021

Published: 17 November 2021

Citation:

Wang J, Liu N, Jiang H, Li Q and Xing D (2021) Reactive Oxygen Species in

Anticancer Immunity: A Double-

Edged Sword.

Front. Bioeng. Biotechnol. 9:784612.

doi: 10.3389/fbioe.2021.784612 blocking (ICB) therapy, T cell activation and expansion, as well as the induction of immunogenic cell death. A majority of reports are indicating detrimental effects of ROS, but it is unadvisable to simply scavenge them because of their pleiotropic effects in most occasions (except in T cell activation and expansion where ROS are generally undesirable). Therefore, clinical success will need a clearer illustration of their multi-faced functions, as well as more advanced technologies to tune ROS level with high spatiotemporal control and species-specificity. With such progresses, the efficacy of current immunotherapies will be greatly improved by combining with ROS-targeted therapies.

Keywords: ROS, immunotherapy, macrophage polarization, T cell activation, immunogenic cell death

\section{INTRODUCTION}

Reactive oxygen species (ROS) are a class of highly reactive oxygen-derived chemicals, including hydroxyl radical $(\cdot \mathrm{OH})$, singlet oxygen $\left({ }^{1} \mathrm{O}_{2}\right)$, superoxide anion $\left(\mathrm{O}_{2}{ }^{-{ }^{-}}\right)$, and peroxides. A group of biological reactions, with the oxidative metabolisms within mitochondria being a major source, can generate ROS in human body. Despite being byproducts in many occasions, ROS at suitable concentrations and locations are vital messengers in cellular signaling and can trigger important biosynthetic processes such as the crosslinking of extracellular matrix (Schieber and Chandel, 2014; Zhou et al., 2020). On the other hand, given the high reactivity of ROS that can be harmful to protein, DNA, and lipids, an antioxidant system has been built to maintain the homeostasis of ROS generation and elimination ( $\mathrm{Yu}$ et al., 2020). Under pathological conditions, the delicate balance will be disturbed and usually lead to ROS accumulation and oxidative stress (Aggarwal et al., 2019). In oncology, evidence has linked the increased ROS level with cancer initiation, progression, angiogenesis, and metastasis (Moldogazieva et al., 2018), making ROS elimination a promising strategy for controlling the disease (Zheng et al., 2021). Paradoxically, ROS can also be beneficial for tumor suppression. For example, the expression of many tumor suppressor genes (e.g., p53) is controlled by ROS (Liu et al., 2008; Perillo et al., 2020); many drugs including chemotherapeutic and radiotherapeutic agents kill cancer cells by elevating ROS level; (Ji et al., 2021; Perillo et al., 2020) etc. 
TABLE 1 | Representative studies reporting the ROS-promoted M2 polarization of macrophages.

\begin{tabular}{|c|c|c|c|c|}
\hline Model & Tested markers & ROS modulation & $\begin{array}{l}\text { Mechanisms } \\
\text { of M2 polarization }\end{array}$ & References \\
\hline $\begin{array}{l}\text { Mouse bone marrow-derived } \\
\text { macrophages }\end{array}$ & $\begin{array}{l}\text { M1: CD86, TNF- } \alpha, \text { IL-12; M2: } \\
\text { IL-10, CCL17/18/24 }\end{array}$ & $\begin{array}{l}\mathrm{O}_{2}{ }^{\bullet-} \text { increment by } \mathrm{NOX} \text {; elimination } \\
\text { by } \mathrm{BHA}\end{array}$ & $\begin{array}{l}\text { ROS induce late-phase activation of ERK } \\
\text { signaling }\end{array}$ & $\begin{array}{l}\text { Zhang, et al. } \\
\text { (2013) }\end{array}$ \\
\hline $\begin{array}{l}\text { Mouse RAW } 264.7 \\
\text { macrophages }\end{array}$ & $\begin{array}{l}\text { M1: CD11b; M2: CD206, } \\
\text { Arg-1 }\end{array}$ & mtROS; reduction by antioxidant & $\begin{array}{l}\text { Antioxidant reduce M2 type via ROS/ERK } \\
\text { and mTOR pathway }\end{array}$ & $\begin{array}{l}\text { Shan, et al. } \\
(2017)\end{array}$ \\
\hline Primary human macrophages & $\begin{array}{l}\text { M1: TNF- } \alpha, \text { IL12b; M2: } \\
\text { CD163, CD206 }\end{array}$ & $\begin{array}{l}\text { Increased via } \mathrm{H}_{2} \mathrm{O}_{2} \text { addition; } \\
\text { reduced using } \mathrm{MnTe}\end{array}$ & $\begin{array}{l}\text { Presumably induce Stat3 activation for M2 } \\
\text { polarization }\end{array}$ & $\begin{array}{l}\text { Griess, et al. } \\
(2020)\end{array}$ \\
\hline $\begin{array}{l}\text { Monocytes in human peripheral } \\
\text { blood mononuclear cells }\end{array}$ & $\begin{array}{l}\text { M1: not tested; M2: CD163, } \\
\text { CD206 }\end{array}$ & $\begin{array}{l}\text { Increment via CAF stimulation; } \\
\text { reduction by } \mathrm{BHA}\end{array}$ & Not directly tested & $\begin{array}{l}\text { Zhang, et al. } \\
(2017)\end{array}$ \\
\hline $\begin{array}{l}\text { Mouse bone marrow-derived } \\
\text { macrophages }\end{array}$ & $\begin{array}{l}\text { M1: IL-6; M2: Arg-1, Mrc1, } \\
\text { IL-10, Ym2, Fizz1 }\end{array}$ & $\begin{array}{l}\text { mtROS increment via GMFG } \\
\text { knockdown; reduction by } \\
\text { antioxidant }\end{array}$ & $\begin{array}{l}\text { Increased mtROS presumably alters iron } \\
\text { metabolism-related protein expression }\end{array}$ & $\begin{array}{l}\text { Aerbajinai, et al. } \\
\text { (2019) }\end{array}$ \\
\hline Murine peritoneal macrophage & $\begin{array}{l}\text { M1: IL-6, TNF- } \alpha \text {, IL-1 } \beta \text {; M2: } \\
\text { Arg-1, Ym1, Fizz1-Relm- } \alpha\end{array}$ & MCPIP-stimulated ROS production & $\begin{array}{l}\text { ROS induced ER stress and autophagy to } \\
\text { increase M2 markers }\end{array}$ & $\begin{array}{l}\text { Kapoor, et al. } \\
\text { (2015) }\end{array}$ \\
\hline
\end{tabular}

NOX: NADPH, oxidase; Arg-1: arginase-1; mtROS: mitochondria ROS; mTOR: mammalian target of rapamycin; MnTe: MnTE-2-PyP(+; Stat3: signal transducer and activator of transcription 3; CAF: cancer-associated fibroblasts; GMFG: glia maturation factor- $\lambda$; MCPIP: MCP-1-induced protein.

Cancer immunotherapy strengthens one's own immune system to recognize and attack tumor cells. The last decade has witnessed the rapid development of immunotherapy with tens of different therapeutics at various treatment modalities been approved by regulatory administrations for clinical use (Smyth et al., 2016; Waldman et al., 2020; Yang et al., 2021). Interestingly, ROS play multiple roles in immunity and can be explored as potent targets to augment the magnitude and specificity of antitumor response (Kotsafti et al., 2020). A large number of studies have reported the benefits of ROS in anticancer immunity; however, the paradox still exists. The often-encountered immunosuppression, such as the attenuated $\mathrm{T}$ cell activation and activity (Qu et al., 2013), raises a necessity for researchers to build a clearer illustration about which role will ROS play under a given condition. This review summarizes recent studies reporting ROS-mediated enhancement or attenuation of antitumor immunity, with an expectation of providing basic rationales for improved immunotherapy.

\section{INTERLACING ROLES OF ROS IN IMMUNOTHERAPY}

Among the multiple fields ROS are functioning, the following four are of particular significance.

\section{Macrophage Polarization}

Macrophages play critical roles in tissue homeostasis by regulating tissue development, mediating inflammatory responses and clearing pathogens and cell debris (DeNardo and Ruffell, 2019; Zheng et al., 2021). They are inducible in function, with the classically activated M1 type exerting proinflammatory and antitumor activities while the M2 type functioning basically the opposite (Mills et al., 2016). Local ROS concentration has an obvious influence on the polarization of macrophages, and based on current evidences ROS may induce pro-inflammatory macrophages more dominantly than doing the opposite. They can activate nuclear factor $\kappa B \quad(\mathrm{NF} \kappa \mathrm{B})$ and p38 mitogen-activated protein kinase (MAPK) signaling pathways and promote the expression of M1-associated pro-inflammatory cytokines and chemokines (Rendra, et al., 2019). This mechanism is widely accepted in innate immunity and has also been reported to augment the antitumor immunity. For example, iron overload, which rapidly induced ROS production, polarized macrophages to pro-inflammatory phenotype, enhanced the activity of p300/CBP acetyltransferase and improved $p 53$ acetylation (Zhou et al., 2018). However, other studies indicated the M2promoting function of ROS. Typical studies involving diverse M2-promiting mechanisms have been summarized in Table 1.

Therefore, ROS can induce the differentiation of macrophages to both M1 and M2 types, raising uncertainty for the direction of ROS modulation (Rendra et al., 2019; Zhou et al., 2020). What further complicates the situation is that factors inducing M1 polarization may not provide benefits for cancer suppression. For example, it was shown that black raspberries, which served as an antioxidant, reduced the incidence of esophageal cancer by suppressing oxidative stress and NFKB/MAPK signaling (Shi, et al., 2017). Given the presence of pathways that lead to contrary results, it can be envisioned that ROS may simultaneously exert opposite influences on macrophages, and the ultimate impact may depend on ROS concentration (including the relative concentration of different species), location and their interaction with therapeutic agents. It is noteworthy that besides M1/M2 polarization, ROS influence macrophages in many other aspects. Roux et al. showed that ROS mediated the immunosuppression effect of macrophages by up-regulating the expression of programmed death ligand-1 (PDL1) (Roux et al., 2019). When treated with ROS inducers such as paclitaxel, PD-L1 expression was up-regulated on the surface of tumor-associated (TAMs) in a mouse model of triple negative breast cancer, via the activation of NFkB signaling. Note that both M1 and M2 signatures positively correlated with the expression of PD-L1. 
Efficacy of Immune Checkpoint Blockades Using monoclonal antibodies to block the immune checkpointmediated immune escape has been one of the most promising strategy for tumor control (Havel et al., 2019). The immune microenvironment exerts a great influence on the treatment efficacy, while ROS serve as critical mediators. A large number of studies have shown that ROS generation would lead to augmented expression of the programmed death-ligand 1 (PDL1) on cancer cells. A straight forward mechanism is that ROS elevation leads to the upregulation or stabilization of multiple

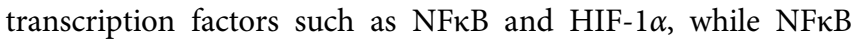
initiates PD-L1 expression by binding to the PD-L1 promoter (Guo et al., 2019), and HIF- $1 \alpha$ directly binds to a transcriptionally active hypoxia-response element in the PD-L1 proximal promoter (Noman et al., 2014). Note that hypoxia-induced HIF- $1 \alpha$ activation can either elevate ROS level via NOX or reduce ROS by inhibiting the tricarboxylic acid cycle (Chen et al., 2018). There are also studies reporting enhanced ROS generation with reduced PD-L1 expression or vice versa, as summarized in a review article (Bailly, 2020). Nevertheless, in most occasions the PD-L1 expression is positively correlated with ROS level, as demonstrated by using a large variety of ROSmodulating small molecules and human oncoviruses (Montani, et al., 2018). Meanwhile, the role macrophage plays in the connection between ROS and PD-L1 expression is worth noting. ROS is a critical mediator of macrophage polarization, while PD-L1 high expression has been found to be correlated with M2-polarization of macrophages (Zhu, et al., 2020). Therefore, skewing the M1/M2 balance of macrophages may be a potential route by which ROS modulate PD-L1 expression.

ROS also have an impact on programmed death-1 (PD-1) blocking therapy at least due to the ligand/receptor relationship of PD-L1/PD-1. Chamoto et al. reported that tumor-reactive T cells boosted by PD-L1 blockade possessed activated mitochondria with augmented ROS production, and improving ROS generation using ROS precursors or mitochondrial uncouplers synergized the antitumor activity of PD-1 blockade by expansion of effector/ memory cytotoxic $\mathrm{T}$ cells in draining lymph nodes (Chamoto et al., 2017). A more recent study reported similar results, that in a "bilateral tumor model", ROS increment in $\mathrm{CD}^{+} \mathrm{T}$ cells was observed only in tumors that were responsive to $\mathrm{PD}-1$ blockade therapy (Kumar et al., 2020). Therefore, ROS level in tumoral and lymphatic cells might be a potential indicator of the responsiveness to $\mathrm{PD}-1 / \mathrm{PD}-\mathrm{L} 1$ blockade therapy, especially considering that the expression of immune checkpoints has been accepted as a tumor-intrinsic sign of the vulnerability of tumors to ICB therapy (Zappasodi et al., 2018).

Cytotoxic T lymphocyte-associated antigen-4 (CTLA-4) is a vital regulator of $\mathrm{T}$ cell function. Studies directly exploring the effect of ROS on CTLA-4 therapy are rare to the best of our knowledge, but ROS have an obvious influence on the development of $\mathrm{T}$ regulatory cells (Tregs), on which CTLA-4 is constitutively expressed (Walker 2013). The effects are still multi-faced. For example, macrophage-generated ROS were functional for the induction of Tregs (Kraaij et al., 2010), while neutrophil cytosolic factor 1-deficient mice with a lower level of ROS also carried Tregs more reactive than those from wild mice (Kim et al., 2014). Obviously, the expansion of CTLA-4 blocking therapy warrants further studies on the effects of ROS.

\section{T Cell Activation and Expansion}

Activation of $\mathrm{T}$ cells, as the pivotal step in cellular immunity, relies on the binding with main histocompatibility complex, the stimulation by co-stimulatory molecules on antigen-presenting cells, and a suitable biochemical environment that allows these processes to happen. ROS have a great influence on $\mathrm{T}$ cell activation. An example is that the local number and phenotype of macrophages, which often function as antigen presenting cells, can be re-/programmed by ROS as described above. Another aspect is the ROS-tuned expression of immune checkpoint molecules on immune cells and cancer cells. More directly, ROS can create an oxidative environment to inactivate $\mathrm{T}$ cells. It was reported that the redox level on cell surface physically determines the reactivity of $\mathrm{T}$ cells (Sahaf et al., 2003). Researchers found that mice were more susceptible to develop severe arthritis if ROS production was diminished, and then revealed that lower ROS level would increase the number of reduced thiol groups on $\mathrm{T}$ cell membrane surface and make T cells more prone to be activated (Gelderman et al., 2006). This is a necessary mechanism to prevent the over-activation of $\mathrm{T}$ cells in inflammatory sites, while for cancer treatment this is commonly undesirable (Moro-Garcia et al., 2018; Yin et al., 2021). Artificially increasing cell surface thiol by adding antioxidants (e.g., glutathione, GSH) or reducing ROS generation has been employed to increase the sensitivity of $\mathrm{T}$ cells to stimulatory signals (Kesarwani, et al., 2013).

Efficient expansion of tumor-specific T cells upon activation is necessary in cell therapy, and the failure to do so has been a major limitation for adoptive cell therapy to achieve broader application. It was shown that the persistence of effector $\mathrm{CD}^{+}$ $\mathrm{T}$ cells and CD62 $\mathrm{L}^{\text {hi }}$ central memory T cells were obviously longer if the cytosolic GSH and surface thiol were higher (Kesarwani et al., 2015), while GSH depletion prevented T cell proliferation despite the stimulation using antigens (Mak et al., 2017). Pretreatment with antioxidant $\mathrm{N}$-acetyl cysteine (NAC) during ex vivo $\mathrm{T}$ cell expansion process significantly improved the persistence of adoptively transferred cells and led to more effective tumor control in a mouse model of melanoma (Scheffel et al., 2016). The underlying mechanism was revealed to be reduction in DNA damage by reducing ROS and the resultant reduced activation-induced cell death (an immunosuppressive process known to be induced by repeated stimulation of T cell receptor) of T cells in the presence of NAC (Scheffel et al., 2016). All these evidence suggests the necessity of adding antioxidants to the culture media of therapeutic $\mathrm{T}$ cell survival and expansion. Note that both GSH and NAC contain thiol groups as potent reducing moiety to scavenge electrons from highly reactive molecules, e.g., to consume ROS.

\section{Immunogenic Cell Death}

Cancer develops with mutations, resulting in the emergence of abundant neoepitopes (sequence-altered nucleic acids and proteins) that are foreign to host's immune system. Immune responses induced by a specific neoepitope may fail to damage 
tumor cells that do not contain this neopitope, while immunogenic cell death (ICD), which is featured by the release of tumor-associated antigens and danger-associated molecular patterns, will provide a full spectrum of neoepitopes to eliminate immune escape caused by tumor heterogeneity. ROS generally have an inducing effect to ICD occurrence. The induction of endoplasmic reticulum (ER) stress, surface exposure of calreticulin, and secretion of adenosine triphosphate (ATP), high-mobility group box 1 (HMGB1) and heat shock protein 70 (HSP70) are requisites for ICD (Bugaut et al., 2013; Van Loenhout et al., 2020), while many of these processes can be triggered by ROS. An example is that bleomycin (an anticancer drug relying on its ability to generate ROS) induced ER stress and autophagy, which then led to calreticulin exposure and release of HMGB1 and ATP to trigger ICD (Bugaut et al., 2013). Actually, many chemotherapeutic small molecules known to kill cancer cells via ROS generation are undergoing clinical trials as ICD inducers beyond chemo drugs, such as doxorubicin, bortezomib, and epirubicin (Vanmeerbeek et al., 2020). Other kinds of agents are also under exploration. For example, a fluorinated mitochondria-disrupting helical polypeptides, which could destabilize mitochondrial outer membrane, was developed to over-produce intracellular ROS (iROS), induce ICD and enhance PD-L1 blockade therapy (Jeong et al., 2021).

Particularly, a number of treatment modalities have intrinsic capability to induce ICD by producing ROS or other critical stimulators. 1) Photodynamic therapy (PDT), which kills cancer cells by generating abundant ROS with the assistance of photosensitizers and light irradiation, can induce ICD and antitumor immunity (Panzarini et al., 2013). Cellular internalization of photosensitizers causes high iROS level and ER stress especially when photosensitizers localizes near the ER. Using a ER-associated photosensitizer, hypericin, Garg et al. found that PDT generated obvious ER stress, and caused cancer cells to secrete ATP, display damage-associated molecular patterns on cell surface and undergo immunogenic apoptosis (Garg et al., 2012; Garg et al., 2012). The display of calreticulin was crucial by providing the motifs needed for the engulfment of PDT-treated cells by dendritic cells (Garg et al., 2012). Using other photosensitizers other than hypericin failed to induce the exposure of calreticulin on cell surface (Garg et al., 2012), suggesting the necessity of choosing suitable photosensitizers or choosing suitable drug carriers to afford enhanced affinity to ER. 2) Sonodynamic therapy is similar with PDT but employs ultrasound as the energy source (Li et al., 2021), and has been reported to elicit ICD. For instance, a nanocomposite loaded with chlorin e6 (as a sonosensitizer) induced ICD via receptor-interacting protein kinase 3-dependent cell necroptosis (Um et al., 2020). 3) Radiotherapy produces ROS via radiolysis and induce ICD, although the break of double-strand DNA was previously considered as the primary mechanism of tumor suppression in radiotherapy. Actually, ICD-mediated antitumor immunity has been recognized as the origin of abscopal effect in radiotherapy.

There are also studies reporting obvious inhibition of ICDinduced immune response by elevated ROS. Kazama et al. reported that ROS would neutralize the stimulatory capacity of dying cells (Kazama et al., 2008). The results showed that caspase activation against mitochondria promoted immune tolerance of apoptotic cells by generating ROS to oxidize the HMGB1 (Kazama et al., 2008). HMGB1 potently act on dendritic cells to stimulate immunity (Dumitriu et al., 2005), so its inactivation promotes immune tolerance. Using a ROS scavenger to consume extracellular ROS (eROS) enhanced the stimulatory effect of dying cells by avoiding the oxidation of HMGB1 (Deng et al., 2020). Therefore, as depicted in Figure 1, there might be a need to induce ER stress via iROS and simultaneously eliminate eROS to avoid the oxidization of the exposed calreticulin and the released stimulatory molecules.

\section{CONCLUSIONS AND PERSPECTIVES}

ROS are continuously generated in a large variety of biochemical reactions. Although a majority of studies are linking them to disease states such as insulin resistance, inflammation, and cancer, ROS play important roles in immune responses. This warrants a very clear understanding of the multi-faced but tunable roles of ROS. There may be more studies reporting detrimental effects of ROS on antitumor immunity than those indicating beneficial effects, since they can drive macrophages to polarize to immunosuppressive types, promote the expression of PD-L1, attenuate the efficacy of ICB therapy, deactivate T cells and inhibit the occurrence of ICD. However, it is not wise to simply scavenge ROS because they have pleiotropic effects in most cases, and also because the detrimental/beneficial switch can be easily shifted by modulating ROS concentration, location, species, and the scenarios they are in. For example, ROS can increase the expression of PD-L1, but it is unachievable to eliminate PD-L1 by scavenging ROS and doing so will greatly attenuate the immuno-stimulatory effects of ROS and cause redox imbalance-related adverse effects. Therefore, clinical application of directly tuning ROS level still has a long way to go.

Meanwhile, most of the reported works have studied ROS as a whole without distinguishing their species, possibly due to the limited specificity of detection probes (e.g., 2',7'dichlorodihydrofluorescein) to ROS species (which include $\cdot \mathrm{OH}, \mathrm{O}_{2}{ }^{--}$and $\mathrm{H}_{2} \mathrm{O}_{2}$ ). Free radicals $\left(\cdot \mathrm{OH}\right.$ and $\mathrm{O}_{2}{ }^{--}$) can readily trigger chain reactions and produce various carbon-centered radicals, while $\mathrm{H}_{2} \mathrm{O}_{2}$ are relatively long-lived and inactive compared with free radicals and commonly exert oxidative capability with the assistance of metal ions such as iron and copper. Such a chemical basis provides a good reason to consider that different species will cause varied magnitude of oxidative stress and mediate distinct signaling pathways (Collin, 2019). The location of the studied ROS is another parameter being important but easily ignored. For example, ROS-producing nanomaterials are widely employed to treat cancer, while the main location (e.g., intracellular or extracellular; intra-lysosomal or intracytoplasmic) is hard to determine since the cellular internalization rate and lysosomal escape efficiency of nanomaterials are difficult to quantify. In this context, choosing biomaterials as ROS inducers with well-defined pharmacokinetics will help. With 


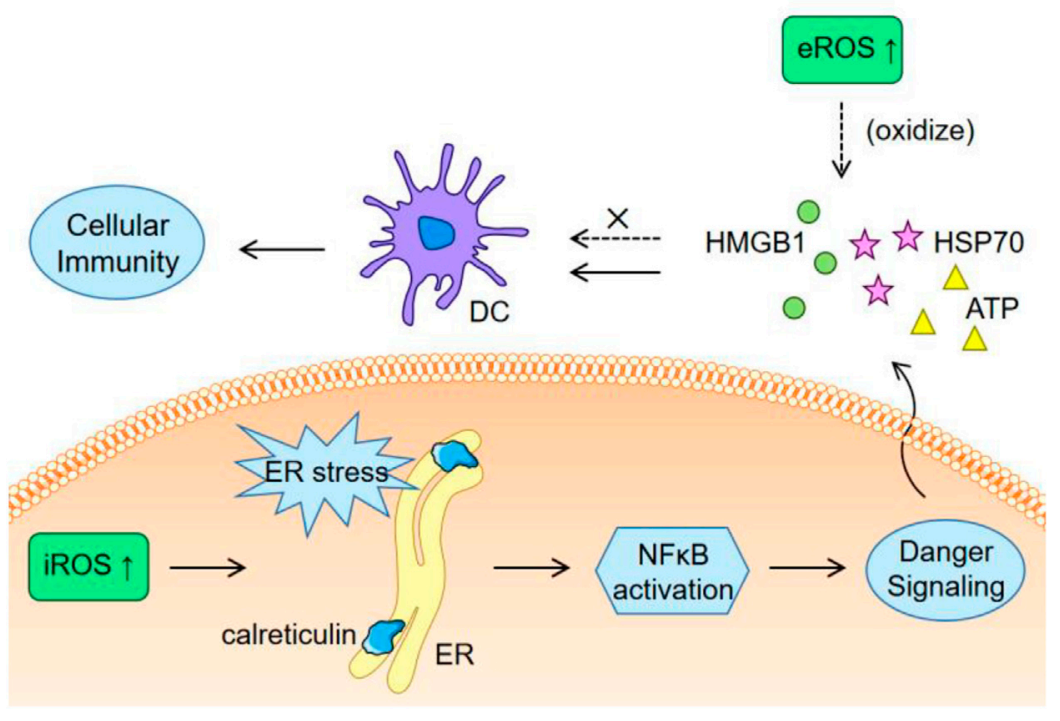

FIGURE 1 | Schematic illustration of the different effects of iROS and eROS on ICD.

the building of such theoretical rationales and technical capabilities, ROS-targeted therapy will eventually synergize with current immunotherapies.

\section{AUTHOR CONTRIBUTIONS}

JW and NL: conceptualization. JW and HJ: drafting. QL and DX: editing and revision. All authors contributed to the article and approved the submitted version.

\section{REFERENCES}

Aerbajinai, W., Ghosh, M. C., Liu, J., Kumkhaek, C., Zhu, J., Chin, K., et al. (2019). Glia Maturation Factor- $\gamma$ Regulates Murine Macrophage Iron Metabolism and M2 Polarization through Mitochondrial ROS. Blood Adv. 3, 1211-1225. doi:10.1182/bloodadvances.2018026070

Aggarwal, V., Tuli, H., Varol, A., Thakral, F., Yerer, M., Sak, K., et al. (2019). Role of Reactive Oxygen Species in Cancer Progression: Molecular Mechanisms and Recent Advancements. Biomolecules 9, 735. doi:10.3390/biom9110735

Bailly, C. (2020). Regulation of PD-L1 Expression on Cancer Cells with ROSModulating Drugs. Life Sci. 246, 117403. doi:10.1016/j.lfs.2020.117403

Bugaut, H., Bruchard, M., Berger, H., Derangère, V., Odoul, L., Euvrard, R., et al. (2013). Bleomycin Exerts Ambivalent Antitumor Immune Effect by Triggering Both Immunogenic Cell Death and Proliferation of Regulatory T Cells. PLoS One 8, e65181. doi:10.1371/journal.pone.0065181

Chamoto, K., Chowdhury, P. S., Kumar, A., Sonomura, K., Matsuda, F., Fagarasan, S., et al. (2017). Mitochondrial Activation Chemicals Synergize with Surface Receptor PD-1 Blockade for T Cell-dependent Antitumor Activity. Proc. Natl. Acad. Sci. USA 114, E761-E770. doi:10.1073/pnas.1620433114

Chen, R., Lai, U. H., Zhu, L., Singh, A., Ahmed, M., and Forsyth, N. R. (2018). Reactive Oxygen Species Formation in the Brain at Different Oxygen Levels: The Role of Hypoxia Inducible Factors. Front. Cel Dev. Biol. 6, 132. doi:10.3389/ fcell.2018.00132

Collin, F. (2019). Chemical Basis of Reactive Oxygen Species Reactivity and Involvement in Neurodegenerative Diseases. Int. J. Mol. Sci. 20, 2407. doi:10.3390/ijms20102407

\section{FUNDING}

This work was financially supported by China Postdoctoral Science Foundation (2021T140355), Postdoctoral Innovation Project of Shandong Province (202002025), the Youth Innovation Team Talent Introduction Program of Shandong Province (20190164), the Qingdao Major Scientific and Technological Project for Distinguished Scholars (20170103).

DeNardo, D. G., and Ruffell, B. (2019). Macrophages as Regulators of Tumour Immunity and Immunotherapy. Nat. Rev. Immunol. 19, 369-382. doi:10.1038/ s41577-019-0127-6

Deng, H., Yang, W., Zhou, Z., Tian, R., Lin, L., Ma, Y., et al. (2020). Targeted Scavenging of Extracellular ROS Relieves Suppressive Immunogenic Cell Death. Nat. Commun. 11, 4951. doi:10.1038/s41467-020-18745-6

Dumitriu, I. E., Baruah, P., Valentinis, B., Voll, R. E., Herrmann, M., Nawroth, P. P., et al. (2005). Release of High Mobility Group Box 1 by Dendritic Cells Controls T Cell Activation via the Receptor for Advanced Glycation End Products. J. Immunol. 174, 7506-7515. doi:10.4049/jimmunol.174.12.7506

Garg, A. D., Krysko, D. V., Vandenabeele, P., and Agostinis, P. (2012). Hypericinbased Photodynamic Therapy Induces Surface Exposure of Damage-Associated Molecular Patterns like HSP70 and Calreticulin. Cancer Immunol. Immunother. 61, 215-221. doi:10.1007/s00262-011-1184-2

Garg, A. D., Krysko, D. V., Verfaillie, T., Kaczmarek, A., Ferreira, G. B., Marysael, T., et al. (2012). A Novel Pathway Combining Calreticulin Exposure and ATP Secretion in Immunogenic Cancer Cell Death. EMBO J. 31, 1062-1079. doi:10.1038/emboj.2011.497

Gelderman, K. A., Hultqvist, M., Holmberg, J., Olofsson, P., and Holmdahl, R. (2006). T Cell Surface Redox Levels Determine T Cell Reactivity and Arthritis Susceptibility. Proc. Natl. Acad. Sci. 103, 12831-12836. doi:10.1073/ pnas.0604571103

Gilardini Montani, M. S., Santarelli, R., Falcinelli, L., Gonnella, R., Granato, M., Di Renzo, L., et al. (2018). EBV Up-regulates PD-L1 on the Surface of Primary Monocytes by Increasing ROS and Activating TLR Signaling and STAT3. J. Leukoc. Biol. 104, 821-832. doi:10.1002/JLB.2A0118-029RR

Griess, B., Mir, S., Datta, K., and Teoh-Fitzgerald, M. (2020). Scavenging Reactive Oxygen Species Selectively Inhibits M2 Macrophage Polarization and Their 
Pro-tumorigenic Function in Part, via Stat3 Suppression. Free Radic. Biol. Med. 147, 48-60. doi:10.1016/j.freeradbiomed.2019.12.018

Guo, R., Li, Y., Wang, Z., Bai, H., Duan, J., Wang, S., et al. (2019). Hypoxiainducible Factor- $1 \alpha$ and Nuclear factor- $\mathrm{\kappa B}$ Play Important Roles in Regulating Programmed Cell Death Ligand 1 Expression by Epidermal Growth Factor Receptor Mutants in Non-small-cell Lung Cancer Cells. Cancer Sci. 110, 1665-1675. doi:10.1111/cas.13989

Havel, J. J., Chowell, D., and Chan, T. A. (2019). The Evolving Landscape of Biomarkers for Checkpoint Inhibitor Immunotherapy. Nat. Rev. Cancer 19, 133-150. doi:10.1038/s41568-019-0116-x

Jeong, S. D., Jung, B. K., Ahn, H. M., Lee, D., Ha, J., Noh, I., et al. (2021). Immunogenic Cell Death Inducing Fluorinated Mitochondria-Disrupting Helical Polypeptide Synergizes with PD-L1 Immune Checkpoint Blockade. Adv. Sci. 8, 2001308. doi:10.1002/advs.202001308

Ji, Y., Han, Z., Ding, H., Xu, X., Wang, D., Zhu, Y., et al. (2021). Enhanced Eradication of Bacterial/Fungi Biofilms by Glucose Oxidase-Modified Magnetic Nanoparticles as a Potential Treatment for Persistent Endodontic Infections. ACS Appl. Mater. Inter. 13, 17289-17299. doi:10.1021/acsami.1c01748

Kapoor, N., Niu, J., Saad, Y., Kumar, S., Sirakova, T., Becerra, E., et al. (2015). Transcription Factors STAT6 and KLF4 Implement Macrophage Polarization via the Dual Catalytic powers of MCPIP. J. Imunol. 194, 6011-6023. doi:10.4049/jimmunol.1402797

Kazama, H., Ricci, J.-E., Herndon, J. M., Hoppe, G., Green, D. R., and Ferguson, T. A. (2008). Induction of Immunological Tolerance by Apoptotic Cells Requires Caspase-dependent Oxidation of High-Mobility Group Box-1 Protein. Immunity 29, 21-32. doi:10.1016/j.immuni.2008.05.013

Kesarwani, P., Murali, A. K., Al-Khami, A. A., and Mehrotra, S. (2013). Redox Regulation of T-Cell Function: From Molecular Mechanisms to Significance in Human Health and Disease. Antioxid. Redox Signaling 18, 1497-1534. doi:10.1089/ars.2011.4073

Kesarwani, P., Thyagarajan, K., Chatterjee, S., Palanisamy, V., and Mehrotra, S. (2015). Anti-oxidant Capacity and Anti-tumor T Cell Function: A Direct Correlation. Oncoimmunology 4, e985942. doi:10.4161/2162402X.2014.985942

Kim, H.-R., Lee, A., Choi, E.-J., Hong, M.-P., Kie, J.-H., Lim, W., et al. (2014). Reactive Oxygen Species Prevent Imiquimod-Induced Psoriatic Dermatitis through Enhancing Regulatory T Cell Function. PLoS One 9, e91146. doi:10.1371/journal.pone.0091146

Kotsafti, A., Scarpa, M., Castagliuolo, I., and Scarpa, M. (2020). Reactive Oxygen Species and Antitumor Immunity-From Surveillance to Evasion. Cancers 12, 1748. doi:10.3390/cancers 12071748

Kraaij, M. D., Savage, N. D. L., van der Kooij, S. W., Koekkoek, K., Wang, J., van den Berg, J. M., et al. (2010). Induction of Regulatory T Cells by Macrophages Is Dependent on Production of Reactive Oxygen Species. Proc. Natl. Acad. Sci. USA 107, 17686-17691. doi:10.1073/pnas.1012016107

Kumar, A., Chamoto, K., Chowdhury, P. S., and Honjo, T. (2020). Tumors Attenuating the Mitochondrial Activity in $\mathrm{T}$ Cells Escape from PD-1 Blockade Therapy. eLife 9, e52330. doi:10.7554/eLife.52330

Li, Y., Xie, J., Um, W., You, D. G., Kwon, S., Zhang, L., et al. (2021). Sono/ Photodynamic Nanomedicine-Elicited Cancer Immunotherapy. Adv. Funct. Mater. 31, 2008061. doi:10.1002/adfm.202008061

Liu, B., Chen, Y., and St. Clair, D. K. (2008). ROS and P53: a Versatile Partnership. Free Radic. Biol. Med. 44, 1529-1535. doi:10.1016/j.freeradbiomed.2008.01.011

Mak, T. W., Grusdat, M., Duncan, G. S., Dostert, C., Nonnenmacher, Y., Cox, M., et al. (2017). Glutathione Primes T Cell Metabolism for Inflammation. Immunity 46, 675-689. doi:10.1016/j.immuni.2017.03.019

Mills, C. D., Lenz, L. L., and Harris, R. A. (2016). A Breakthrough: MacrophageDirected Cancer Immunotherapy. Cancer Res. 76, 513-516. doi:10.1158/00085472.CAN-15-1737

Moldogazieva, N. T., Lutsenko, S. V., and Terentiev, A. A. (2018). Reactive Oxygen and Nitrogen Species-Induced Protein Modifications: Implication in Carcinogenesis and Anticancer Therapy. Cancer Res. 78, 6040-6047. doi:10.1158/0008-5472.CAN-18-0980

Moro-García, M. A., Mayo, J. C., Sainz, R. M., and Alonso-Arias, R. (2018). Influence of Inflammation in the Process of $\mathrm{T}$ Lymphocyte Differentiation: Proliferative, Metabolic, and Oxidative Changes. Front. Immunol. 9, 339. doi:10.3389/fimmu.2018.00339

Noman, M. Z., Desantis, G., Janji, B., Hasmim, M., Karray, S., Dessen, P., et al. (2014). PD-L1 Is a Novel Direct Target of HIF-1a, and its Blockade under
Hypoxia Enhanced MDSC-Mediated T Cell Activation. J. Exp. Med. 211, 781-790. doi:10.1084/jem.20131916

Panzarini, E., Inguscio, V., and Dini, L. (2013). Immunogenic Cell Death: Can it Be Exploited in PhotoDynamic Therapy for Cancer? Biomed. Res. Int. 2013, 1-18. doi:10.1155/2013/482160

Perillo, B., Di Donato, M., Pezone, A., Di Zazzo, E., Giovannelli, P., Galasso, G., et al. (2020). ROS in Cancer Therapy: the Bright Side of the Moon. Exp. Mol. Med. 52, 192-203. doi:10.1038/s12276-020-0384-2

Qu, K., Shen, N.-y., Xu, X.-s., Su, H.-b., Wei, J.-c., Tai, M.-h., et al. (2013). Emodin Induces Human T Cell Apoptosis In Vitro by ROS-Mediated Endoplasmic Reticulum Stress and Mitochondrial Dysfunction. Acta Pharmacol. Sin. 34, 1217-1228. doi:10.1038/aps.2013.58

Rendra, E., Riabov, V., Mossel, D. M., Sevastyanova, T., Harmsen, M. C., and Kzhyshkowska, J. (2019). Reactive Oxygen Species (ROS) in Macrophage Activation and Function in Diabetes. Immunobiology 224, 242-253. doi:10.1016/j.imbio.2018.11.010

Roux, C., Jafari, S. M., Shinde, R., Duncan, G., Cescon, D. W., Silvester, J., et al. (2019). Reactive Oxygen Species Modulate Macrophage Immunosuppressive Phenotype through the Up-Regulation of PD-L1. Proc. Natl. Acad. Sci. USA 116, 4326-4335. doi:10.1073/pnas.1819473116

Sahaf, B., Heydari, K., Herzenberg, L. A., and Herzenberg, L. A. (2003). Lymphocyte Surface Thiol Levels. Proc. Natl. Acad. Sci. 100, 4001-4005. doi:10.1073/pnas.2628032100

Scheffel, M. J., Scurti, G., Simms, P., Garrett-Mayer, E., Mehrotra, S., Nishimura, M. I., et al. (2016). Efficacy of Adoptive T-Cell Therapy Is Improved by Treatment with the Antioxidant N-Acetyl Cysteine, Which Limits Activation-Induced T-Cell Death. Cancer Res. 76, 6006-6016. doi:10.1158/0008-5472.CAN-160587

Schieber, M., and Chandel, N. S. (2014). ROS Function in Redox Signaling and Oxidative Stress. Curr. Biol. 24, R453-R462. doi:10.1016/j.cub.2014.03.034

Shan, M., Qin, J., Jin, F., Han, X., Guan, H., Li, X., et al. (2017). Autophagy Suppresses Isoprenaline-Induced M2 Macrophage Polarization via the ROS/ ERK and mTOR Signaling Pathway. Free Radic. Biol. Med. 110, 432-443. doi:10.1016/j.freeradbiomed.2017.05.021

Shi, N., Chen, F., Zhang, X., Clinton, S., Tang, X., Sun, Z., et al. (2017). Suppression of Oxidative Stress and NFKB/MAPK Signaling by Lyophilized Black Raspberries for Esophageal Cancer Prevention in Rats. Nutrients 9, 413. doi:10.3390/nu9040413

Smyth, M. J., Ngiow, S. F., Ribas, A., and Teng, M. W. L. (2016). Combination Cancer Immunotherapies Tailored to the Tumour Microenvironment. Nat. Rev. Clin. Oncol. 13, 143-158. doi:10.1038/ nrclinonc.2015.209

Um, W., Ko, H., You, D. G., Lim, S., Kwak, G., Shim, M. K., et al. (2020). Necroptosis-Inducible Polymeric Nanobubbles for Enhanced Cancer Sonoimmunotherapy. Adv. Mater. 32, 1907953. doi:10.1002/ adma.201907953

Van Loenhout, J., Peeters, M., Bogaerts, A., Smits, E., and Deben, C. (2020). Oxidative Stress-Inducing Anticancer Therapies: Taking a Closer Look at Their Immunomodulating Effects. Antioxidants 9, 1188. doi:10.3390/antiox9121188

Vanmeerbeek, I., Sprooten, J., De Ruysscher, D., Tejpar, S., Vandenberghe, P., Fucikova, J., et al. (2020). Trial Watch: Chemotherapy-Induced Immunogenic Cell Death in Immuno-Oncology. Oncoimmunology 9, 1703449. doi:10.1080/ 2162402X.2019.1703449

Waldman, A. D., Fritz, J. M., and Lenardo, M. J. (2020). A Guide to Cancer Immunotherapy: from T Cell Basic Science to Clinical Practice. Nat. Rev. Immunol. 20, 651-668. doi:10.1038/s41577-020-0306-5

Walker, L. S. K. (2013). Treg and CTLA-4: Two Intertwining Pathways to Immune Tolerance. J. Autoimmun. 45, 49-57. doi:10.1016/j.jaut.2013.06.006

Yang, L., Pijuan-Galito, S., Rho, H. S., Vasilevich, A. S., Eren, A. D., Ge, L., et al. (2021). High-Throughput Methods in the Discovery and Study of Biomaterials and Materiobiology. Chem. Rev. 121, 4561-4677. doi:10.1021/ acs.chemrev.0c00752

Yin, X., Hao, Y., Lu, Y., Zhang, D., Zhao, Y., Mei, L., et al. (2021). BioMultifunctional Hydrogel Patches for Repairing Full-Thickness Abdominal Wall Defects. Adv. Funct. Mater. 31, 2105614. doi:10.1002/adfm.202105614

Yu, Z., Li, Q., Wang, J., Yu, Y., Wang, Y., Zhou, Q., et al. (2020). Reactive Oxygen Species-Related Nanoparticle Toxicity in the Biomedical Field. Nanoscale Res. Lett. 15, 115. doi:10.1186/s11671-020-03344-7 
Zappasodi, R., Wolchok, J. D., and Merghoub, T. (2018). Strategies for Predicting Response to Checkpoint Inhibitors. Curr. Hematol. Malig. Rep. 13, 383-395. doi:10.1007/s11899-018-0471-9

Zhang, A., Qian, Y., Ye, Z., Chen, H., Xie, H., Zhou, L., et al. (2017). Cancerassociated Fibroblasts Promote M2 Polarization of Macrophages in Pancreatic Ductal Adenocarcinoma. Cancer Med. 6, 463-470. doi:10.1002/cam4.993

Zhang, Y., Choksi, S., Chen, K., Pobezinskaya, Y., Linnoila, I., and Liu, Z.-G. (2013). ROS Play a Critical Role in the Differentiation of Alternatively Activated Macrophages and the Occurrence of Tumor-Associated Macrophages. Cell Res 23, 898-914. doi:10.1038/cr.2013.75

Zheng, W., Hao, Y., Wang, D., Huang, H., Guo, F., Sun, Z., et al. (2021). Preparation of Triamcinolone Acetonide-Loaded Chitosan/fucoidan Hydrogel and its Potential Application as an Oral Mucosa Patch. Carbohydr. Polym. 272, 118493. doi:10.1016/j.carbpol.2021.118493

Zheng, W., Zhou, Q., and Yuan, C. (2021). Nanoparticles for Oral Cancer Diagnosis and Therapy. Bioinorganic Chem. Appl. 2021, 1-14. doi:10.1155/ 2021/9977131

Zhou, Q., Chen, J., Luan, Y., Vainikka, P. A., Thallmair, S., Marrink, S. J., et al. (2020). Unidirectional Rotating Molecular Motors Dynamically Interact with Adsorbed Proteins to Direct the Fate of Mesenchymal Stem Cells. Sci. Adv. 6, eaay2756. doi:10.1126/sciadv.aay2756

Zhou, Y., Que, K.-T., Zhang, Z., Yi, Z. J., Zhao, P. X., You, Y., et al. (2018). Iron Overloaded Polarizes Macrophage to Proinflammation Phenotype through ROS/acetyl-p53 Pathway. Cancer Med. 7, 4012-4022. doi:10.1002/cam4.1670
Zhou, Z., Ni, K., Deng, H., and Chen, X. (2020). Dancing with Reactive Oxygen Species Generation and Elimination in Nanotheranostics for Disease Treatment. Adv. Drug Deliv. Rev. 158, 73-90. doi:10.1016/j.addr.2020.06.006

Zhu, Z., Zhang, H., Chen, B., Liu, X., Zhang, S., Zong, Z., et al. (2020). PD-L1Mediated Immunosuppression in Glioblastoma Is Associated with the Infiltration and M2-Polarization of Tumor-Associated Macrophages. Front. Immunol. 11, 588552. doi:10.3389/fimmu.2020.588552

Conflict of Interest: The authors declare that the research was conducted in the absence of any commercial or financial relationships that could be construed as a potential conflict of interest.

Publisher's Note: All claims expressed in this article are solely those of the authors and do not necessarily represent those of their affiliated organizations, or those of the publisher, the editors and the reviewers. Any product that may be evaluated in this article, or claim that may be made by its manufacturer, is not guaranteed or endorsed by the publisher.

Copyright (c) 2021 Wang, Liu, Jiang, Li and Xing. This is an open-access article distributed under the terms of the Creative Commons Attribution License (CC BY). The use, distribution or reproduction in other forums is permitted, provided the original author(s) and the copyright owner(s) are credited and that the original publication in this journal is cited, in accordance with accepted academic practice. No use, distribution or reproduction is permitted which does not comply with these terms. 